\title{
KDM5C Is Overexpressed in Prostate Cancer and Is a Prognostic Marker for Prostate-Specific Antigen-Relapse Following Radical Prostatectomy
}

Johannes Stein, ${ }^{*}$ Michael Majores, ${ }^{*}$ Magdalena Rohde, ${ }^{*}$ Soyoung Lim, ${ }^{\dagger}$ Simon Schneider, ${ }^{*}$ Eliana Krappe, ${ }^{*}$ Jörg Ellinger, Manfred Dietel, ${ }^{\S}$ Carsten Stephan, ${ }^{\complement}$ Klaus Jung, ${ }^{\|}$Sven Perner, ${ }^{*}$ Glen Kristiansen, ${ }^{*}$ and Jutta Kirfel*

From the Institute of Pathology, ${ }^{*}$ Department of Urology, ${ }^{\ddagger}$ University of Bonn, Bonn, Germany; the Institute of Pathology, ${ }^{\dagger}$ University of Cologne, Köln, Germany; and the Institute of Pathology, ${ }^{\S}$ Department of Urology, ${ }^{\circledR}$ and the Berlin Institute of Urologic Research, "Charite University Hospital, Berlin, Germany

Accepted for publication

May 27, 2014.

Address correspondence to Jutta Kirfel, Ph.D., Institute of Pathology, University Hospital Bonn, Sigmund-Freud-Str. 25, D-53127 Bonn, Germany. E-mail: jutta.kirfel@ukb.unibonn.de.

\begin{abstract}
Currently, few prognostic factors are available to predict the emergence of castration-resistant prostate cancer and no curative options are available. Epigenetic gene regulation has been shown to trigger prostate cancer metastasis and androgen independence. Histone lysine demethylases (KDMs) are epigenetic enzymes that can remove both repressive and activating histone marks. KDM5 family members are capable of removing the histone $\mathrm{H} 3$ lysine 4 dimethylation-activating mark, rendering them potential players in the down-regulation of tumor suppressors and suggesting that their activity could repress oncogenes. Here, we systematically investigated KDM5C expression patterns in two independent radical prostatectomy cohorts (822 prostate tumors in total) by immunohistochemistry. Positive nuclear KDM5C staining was significantly associated with a reduced prostate-specific antigen relapse-free survival. Our study confirmed that nuclear KDM5C expression is an independent prognostic parameter. Most strikingly, the prognostic value of nuclear KDM5C expression for progression-free survival was exclusively pronounced for the Gleason group 7. In addition, KDM5C knockdown resulted in growth retardation of prostate cancer cells in vitro and induced regulation of several proliferationassociated genes. Our data indicate that KDM5C is functionally involved in proliferation control of prostate cancer cells and might represent a novel attractive therapy target. Moreover, overexpression of $\mathrm{KDM} 5 \mathrm{C}$ is an independent new predictive marker for therapy failure as determined by biochemical recurrence in patients after prostatectomy. (Am J Pathol 2014, 184: 2430-2437; http://dx.doi.org/ 10.1016/j.ajpath.2014.05.022)
\end{abstract}

In Western countries prostate cancer (PCa) is the most common male neoplasm and the second-leading cause of male cancer-related deaths. ${ }^{1}$ Since the beginning of the screening era for prostate-specific antigen (PSA) the detection rate of $\mathrm{PCa}$ has increased, resulting in a more frequent detection of indolent cancers. Conversely, the increasing incidence of $\mathrm{PCa}$ has reinforced the need to distinguish indolent cases from aggressive tumors. Early PCa can be treated by surgery or radiation. ${ }^{2}$ The only option in metastatic disease is castration through pharmacologic disruption of androgen receptor signaling, which is a major proliferation stimulus for prostate cells. However, after a variable time PCa may evolve into an aggressive castrationresistant $\mathrm{PCa}^{3}$ displaying an 18-month median survival. ${ }^{4}$
Castration-resistant $\mathrm{PCa}$ is resistant to conventional treatments, and chemotherapy itself can only delay its progression. Currently, few molecular targets are available to treat castration-resistant $\mathrm{PCa}$, and few prognostic factors are associated with $\mathrm{PCa}$ recurrence and progression. ${ }^{5}$ Thus, the identification of novel prognostic factors and therapeutic targets for castration-resistant PCa is highly needed.

In the past decade, it has become evident that epigenetic gene regulation plays a crucial role in PCa initiation and

Supported by grants from the Deutsche Forschungsgemeinschaft (J.K.).

G.K. and J.K. contributed equally as senior authors. J.S., M.M., and M.R. contributed equally to this work.

Disclosures: None declared. 
progression. Epigenetic refers to all heritable changes that do not depend on modifications of DNA primary sequence. In 2004, a human lysine-specific demethylase (KDM1 A, LSD1) was described. ${ }^{6}$ Lysine demathylases (KDMs) may counteract different histone methyltransferases (polycomb group or trithorax members), thereby activating or repressing gene expression. Because histone post-translational modifications are known to orchestrate critical functions of PCa cells, it is conceivable that KDMs are key players of those processes. KDM1A, the best characterized lysine-specific demethylase, is a putative oncogene in several neoplasms, including PCa. Because histone $\mathrm{H} 3$ lysine 4 dimethylation ( $\mathrm{H} 3 \mathrm{~K} 4 \mathrm{me} 2$ ) is an active mark, KDM1 A favors gene silencing. However, when recruited by androgen receptor, KDM1A loses its capability to demethylate $\mathrm{H} 3 \mathrm{~K} 4 \mathrm{me} 1 / 2$ and instead catalyzes the demethylation of $\mathrm{H} 3 \mathrm{~K} 9 \mathrm{me} 1 / 2$, two repressive marks, thus acting as a coactivator. ${ }^{7,8}$ Because KDM1A is capable of mono- and di-demethylase activity, a second class of KDMs is represented by the $\mathrm{Fe}^{2+}$ /oxoglutarate-dependent enzymes, containing a characteristic Jumonji C domain. ${ }^{9}$ Those KDMs are divided into six clusters (KDM2 to KDM7). Each cluster is characterized by one or more target histone residues. For example, KDM5 clusters are mainly involved in removing active marks (H3K4me2 and $\mathrm{H} 3 \mathrm{~K} 36 \mathrm{me} 2)$, thereby triggering gene silencing. ${ }^{9}$

Members of the KDM5 family have distinct biological functions; they can be crucial in the expression and repression of oncogenes and tumor suppressor genes and can themselves serve as both. Several studies have shown a direct involvement of KDM5A and KDM5B in cancer. Loss of Kdm5a inhibits tumorigenesis in a mouse model. ${ }^{10}$ In addition, KDM5A is overexpressed in gastric cancer, and high levels of KDM5B have been observed in breast cancer and PCa. ${ }^{11,12}$ KDM5A and KDM5B are involved in maintenance of a slow-growing, drug-resistant population of cancer cells. $^{13,14}$ Another KDM5 family protein, KDM5C (also known as JARID1C and SMCX), has mostly been studied in the context of mental retardation, ${ }^{15}$ but it has been linked to clear cell renal cell carcinomas. As part of the Cancer Genome Project, an extensive analysis of clear cell renal cell carcinoma led to the discovery that $3 \%$ of clear cell renal cell carcinoma tumors contain truncation mutations in KDM5C. ${ }^{16}$

In this study, we aimed to clarify the expression patterns and the biological relevance of KDM5C in PCa with a largescale expression analysis and functional studies.

\section{Materials and Methods}

\section{TMAs}

Tissue microarrays (TMAs) were prepared from formalinfixed, paraffin-embedded tissue specimens of PCas selected from the archival files of the Institute of Pathology Bonn $(n=262)$ and by an additional cohort of the Charite University Hospital Berlin $(n=560)$. Cases were selected according to tissue availability for research purposes without regard to clinical or prognostic parameters. Both carcinoma and nontumorous portions were represented at TMA comprising five tissue samples of $1.2-\mathrm{mm}$ or $1.8-\mathrm{mm}$ diameter for each case and were arrayed from formalin-fixed, paraffin-embedded tissue blocks by using a manual device. The different cohorts are characterized as listed below.

\section{Bonn Cohort}

All tumor samples were surgically obtained from patients who had undergone radical retropubic prostatectomy in the surgical center (University Hospital Bonn) between 1998 and 2008 for clinically organ-confined PCa. Mean age at date of surgery was $63.87 \pm 6.4$ years (range, 45 to 83 years). A total of $166(63.4 \%), 91$ (34.7\%), and 5 (1.9\%) had pathological stage pT2, pT3, and pT4, respectively. Nodal status was positive in 21 cases (8\%) and surgical margins were positive in 70 cases $(27 \%$; cases with unknown nodal status, $n=3$; cases with unknown residual status, $n=5$ ). Because of the long observation period with changes of the criteria of Gleason grading, a senior pathologist reassigned the Gleason score according to the recommendations of the International Society of Urological Pathology Consensus Conference in 2005. ${ }^{17}$ Overall a Gleason score of $<7,7$, and $>7$ was graded for 80 cases $(30.5 \%), 132$ cases $(50.4 \%)$, and 50 cases $(19.1 \%)$, respectively. Follow-up data could be ascertained for 243 patients $(92.7 \%)$. At a mean follow-up period of $87.7 \pm 41.2$ months (range, 0 to 170 months), death was documented in 36 cases (13.7\%) and among 3 cases (1.1\%) death was definitely related to PCa. The PSA relapse status was evaluable for 230 patients (87.8\%). PSA relapse was defined as re-elevated at PSA level $\geq 0.2 \mathrm{ng} / \mathrm{mL}$ after prostatectomy and was documented in 44 cases (19.1\%).

\section{Berlin Cohort}

The investigated TMAs comprised 560 samples of radical prostatectomy specimens after surgery in the Department of Urology (Charité University Hospital Berlin) between 1999 and 2005. Mean age at date of prostatectomy was $61.8 \pm 5.7$ years (range, 43 to 74 years). A count of 384 samples (68.6\%), 175 samples $(31.3 \%)$, and 1 sample $(0.2 \%)$ had pT2, pT3, and pT4 stage, respectively. Surgical margins were positive in 150 cases (26.8\%; cases with unknown residual status, $n=2$ ). The Gleason score distribution of this cohort was as follows: <7, 196 (35\%); 7, 268 (47.9\%); and >7, 96 (17.1\%). Overall, PSA relapse status was evaluable for 531 patients $(94.8 \%)$. PSA relapse was seen in 91 cases $(17.1 \%)$.

\section{Immunohistochemistry}

The paraffin-embedded TMAs were freshly cut into 2- to $3-\mu \mathrm{m}$ sections and mounted to Super Frost Plus Slides. After drying overnight, the sections were deparaffinized and pretreated with citrate buffer $(\mathrm{pH} 6)$ for 20 minutes in a microwave to achieve antigen retrieval. Endogenous 
peroxidase activity was quenched with a hydrogen peroxidase block for 10 minutes. Slides were incubated with the primary antibody (rabbit polyclonal KDM5C ab 34718; dilution 1:1000; Abcam, Cambridge, UK) for 30 minutes at room temperature and were subsequently detected by using the Bright Vision Poly-HRP-anti-rabbit-IgG detection reagent (Immunologic, Duiven, The Netherlands) with diaminobenzidine as the chromogen. The slides were counterstained with hematoxylin and dehydrated. Negative control reactions included replacing the primary specific antibody by nonspecific immunoglobulin (data not shown). The specificity of the antibody was tested by Western blot analyses on recombinant protein (data not shown).

\section{Evaluation of Immunohistochemistry}

Staining intensity was scored semiquantitatively in negative, weak, moderate, or strong staining. Previous studies have identified $\mathrm{KDM} 5 \mathrm{C}$ as a protein shuttling from the cytoplasm into the nucleus with the intracellular trafficking controlled by its association with proliferating cell nuclear antigen (PCNA). ${ }^{18}$ Therefore, KDM5C staining was evaluated separately for cytoplasmic and nuclear stainings.

\section{Cell Culture}

PC3, LnCAP, and DU145 cells were cultured in RPMI 1640 that contained $10 \%$ fetal calf serum, $1 \%$ L-glutamine, and $1 \%$ antibiotics (Life Technologies, Carlsbad, CA). Benign prostatic hyperplastic cells were cultured in RPMI 1640 that contained $20 \%$ fetal calf serum, $1 \%$ L-glutamine, $1 \%$ antibiotics, $5 \mu \mathrm{g} / \mathrm{mL}$ insulin, $20 \mathrm{ng} / \mathrm{mL}$ testosterone, $5 \mu \mathrm{g} / \mathrm{mL}$ transferrin, and $5 \mu \mathrm{g} / \mathrm{mL}$ sodium selenite. Cells were purchased from DSMZ (Braunschweig, Germany).

\section{siRNA Transfection}

Cells were transfected as described in Lim et al. ${ }^{19}$ Control siRNA (Qiagen, Hilden, Germany) or siRNAs against KDM5C (D-010097-01 and D-010097-02;Thermo Scientific Dharmacon, Schwerte, Germany) were used in a range of 10 to $20 \mathrm{nmol} / \mathrm{L}$. To verify knockdown of KDM5C, RNA and cDNA were prepared after the second round of siRNA transfection (6 days).

\section{MTT Assay}

MTT assay was performed, according to the manufacturer's protocol (Roche, Mannheim, Germany).

\section{qPCR}

Total RNA was isolated from cells by using the RNeasy Mini kit (Qiagen), and cDNA synthesis was performed with the SuperScript Reverse Transcription kit (Invitrogen, Carlsbad,
CA). Real-time quantitative PCR (qPCR) was done as described by Lim et al. ${ }^{19}$ Expression values were normalized to the mean of $18 \mathrm{~S}$ rRNA and $\beta$-actin levels. Primers were designed with Universal ProbeLibraryAssayDesignCenter (Roche Applied Science, Minneapolis, MN). Data were analyzed with the $2^{-\Delta \Delta \mathrm{Ct}}$ method. The results are presented as fold change to scrambled siRNA-transfected samples which were set to 1. Error bars indicate SEM. Results present the mean of five independent experiments. Primer sequences were as follows: KDM5C-forward, 5'-GAGGTGACCCTGGATGAGAA-3', and KDM5C-reverse, 5'-CAGGAGCTGAGGTCTGAAC- ${ }^{\prime}$; MKI67-forward, 5'-CAAGACTCGGTCCCTGAAAA-3', and MKI67-reverse, 5'-TTGCTGTTCTGCCTCAGTCTT-3'; PCNA-forward, 5'-TGTCACAGACAAGTAATGTCGATAAA- ${ }^{\prime}$, and PCNA-reverse, $5^{\prime}$-GAACTGGTTCATTCATCTCTATGG-3'; p21-forward, 5'-CGAAGTCAGTTCCTTGTGGAG-3', and p21-reverse, 5'- CATGGGTT-CTGACGGACAT- $3^{\prime}$; 18S-forward, $5^{\prime}$-CGATTGGATGGTTTAGTGAGG-3', and 18S-reverse, 5'-AGTTCGACCGTCTTCCTCAGC-3'; and actin-forward, 5'-CCAACCGCGAGAAGATGA- $3^{\prime}$, and actin-reverse 5'-CCAGAGGCGTACAGGGATAG-3'.

\section{Statistical Analysis}

All statistics were performed with SPSS version 21.0 (SPSS Inc., Chicago, IL). Nuclear KDM5C staining intensity was dichotomized, grouping nuclear positive cases against the negative ones. To assess statistical significance of the associations between nuclear KDM5C expression and clinicopathologic parameters $\chi^{2}$ tests were applied. Univariate survival analyses were calculated with Cox regression and Kaplan-Meier statistic. Log-rank values were used to evaluate differences of survival curves. Multivariate survival analysis was performed on all of the parameters that were found to be significant on univariate analysis by using the Cox regression model. $P<0.05$ was considered significant.

All statistical analyses refer to the total sample of both cohorts with a sample size of $n=822(n=761)$.

\section{Results}

\section{Nuclear and Cytoplasmic Staining of KDM5C}

KDM5C showed a cytoplasmic and also nuclear staining pattern in benign and tumor tissues; however, benign tissue showed only a limited nuclear positivity. Cytoplasmic KDM5C staining was observed in the majority of prostate carcinoma samples (741 cases, $90.2 \%$ ). Weak and moderate cytoplasmic stainings were observed among $386(47 \%)$ and 299 cases (36.4\%), respectively, whereas strong cytoplasmic staining was observed in 56 cases $(6.8 \%)$.

Nuclear KDM5C staining was detected in 246 cases (29.9\%). Weak, moderate, and strong stainings were observed in 134 (16.3\%), 89 (10.8\%), and 23 cases (2.8\%), 

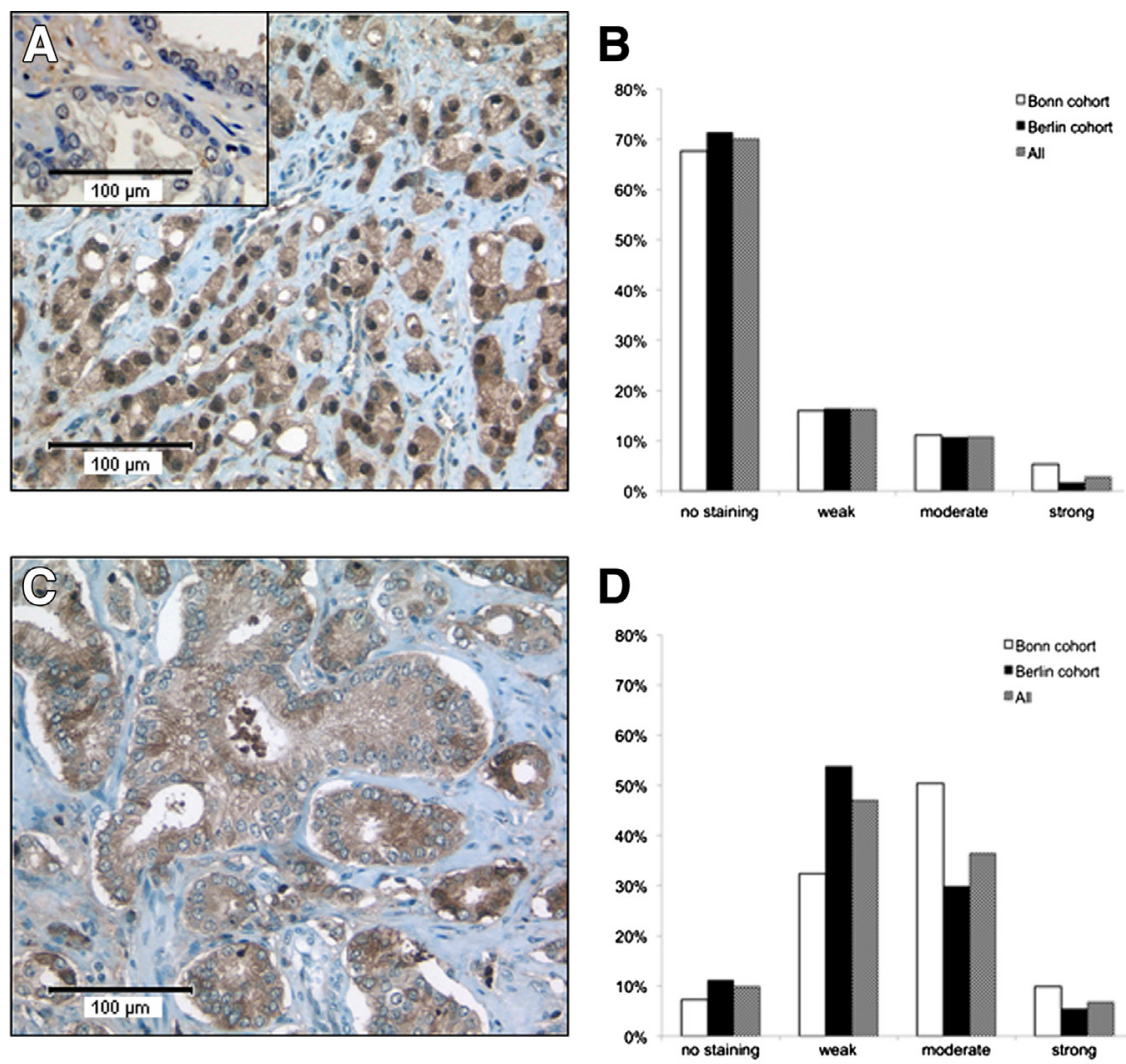

Figure 1 Immunohistochemical staining of KDM5C in PCa. A: Despite frequently detectable cytoplasmic staining of weak or moderate intensity, an additional nuclear staining could be distinguished in a subset of tumors. C: In contrast, other samples only showed an exclusively cytoplasmic (with negative nuclei) staining. Inset in A shows immunohistochemical staining of KDM5C in benign tissue. Bar graphs show the distributions of nuclear (B) and cytoplasmic (D) stainings of the tumors of the cohorts. respectively. Nuclear staining was distributed in a greater proportion to the cells that showed weak ( 87 of $301,28.9 \%$ ) or moderate (59 of $167,35.3 \%$ ) cytoplasmic staining. Only about one-sixth of carcinoma without (10 of $62,16.1 \%$ ) or with (5 of 30,16.7\%) strong cytoplasmic staining showed nuclear staining as well (Figure 1 and Table 1).

\section{Statistical Analyses and Survival Analyses}

Correlation of KDM5C with clinicopathologic data showed strong correlation with pT status (Table 1). As expected, univariate survival analyses (Cox regression) confirmed the residual status, pT status, and Gleason score as significant

Table 1 Clinicopathologic Parameters and Their Associations ( $\chi^{2}$ Test) with Nuclear KDM5C Expression

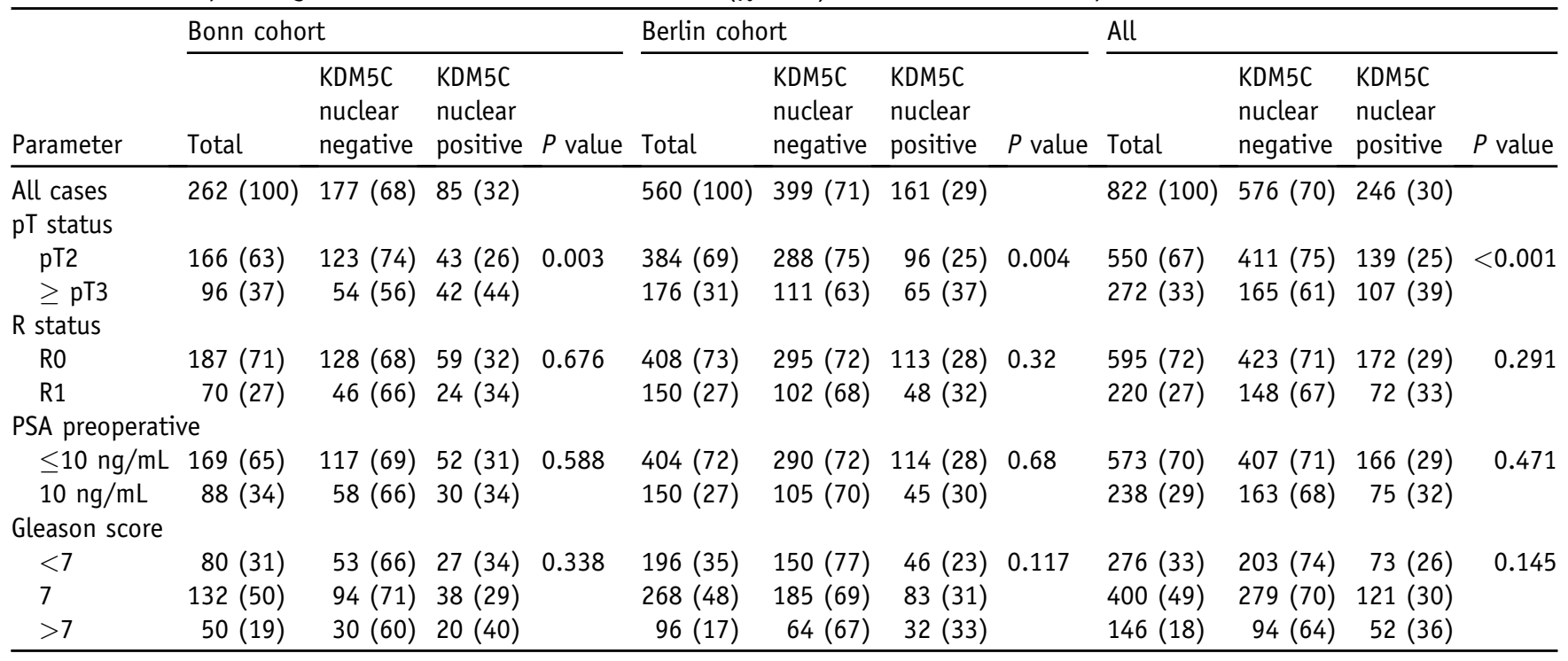

Data are expressed as $n(\%)$. In the Bonn cohort five cases were Rx and in five cases the PSA was not evaluable. In the Berlin cohort two cases were Rx and in six cases PSA was not evaluable.

KDM5C, lysine demethylase 5C; PSA, prostate-specific antigen; PT, pathological stage; R, residual; Rx, unknown residual status. 
Table 2 Univariate Survival Analysis (Cox Regression) Regarding PSA Relapse-Free Survival after Prostatectomy

\begin{tabular}{|c|c|c|c|c|c|c|c|c|c|c|c|c|}
\hline \multirow[b]{2}{*}{ Parameter } & \multicolumn{4}{|c|}{ Bonn cohort } & \multicolumn{4}{|c|}{ Berlin cohort } & \multicolumn{4}{|l|}{ All } \\
\hline & $\begin{array}{l}\text { No. of } \\
\text { cases }\end{array}$ & $\begin{array}{l}\text { No. of } \\
\text { events }\end{array}$ & $P$ value & $\operatorname{Exp}(B)$ & $\begin{array}{l}\text { No. of } \\
\text { cases }\end{array}$ & $\begin{array}{l}\text { No. of } \\
\text { events }\end{array}$ & $P$ value & $\operatorname{Exp}(B)$ & $\begin{array}{l}\text { No. of } \\
\text { cases }\end{array}$ & $\begin{array}{l}\text { No. of } \\
\text { events }\end{array}$ & $P$ value & $\operatorname{Exp}(B)$ \\
\hline Negative & 153 & 23 & 0.007 & 2.295 & 382 & 53 & 0.003 & 1.87 & 535 & 76 & $<0.001$ & 1.998 \\
\hline Positive & 77 & 21 & & & 149 & 38 & & & 226 & 59 & & \\
\hline \multicolumn{13}{|l|}{ R status } \\
\hline \multicolumn{13}{|l|}{ pT status } \\
\hline pT2 & 151 & 20 & 0.003 & 2.435 & 364 & 33 & $<0.001$ & 4.329 & 515 & 53 & $<0.001$ & 3.551 \\
\hline$>$ pT2 & 79 & 24 & & & 167 & 58 & & & 246 & 82 & & \\
\hline \multicolumn{13}{|l|}{ Gleason score } \\
\hline$<7$ & 74 & 2 & $<0.001$ & 3.638 & 190 & 14 & $<0.001$ & 2.446 & 264 & 16 & $<0.001$ & 2.748 \\
\hline$>10 \mathrm{ng} / \mathrm{mL}$ & 75 & 18 & & & 146 & 33 & & & 221 & 51 & & \\
\hline
\end{tabular}

$\operatorname{Exp}(B)$, hazard ratio; KDM5C, lysine demethylase 5C; PSA, prostate-specific antigen; $p T$, pathological stage; $R$, residual.

prognostic parameters (each $P<0.001$ ) (Table 2). These data underlined the representativeness of our cohort for subsequent analyses.

Positive nuclear KDM5C staining was significantly associated with a reduced PSA relapse-free survival $(P<0.001)$ (Table 2 and Figure 2A). Because nuclear KDM5C expression was found more frequently in cases of advanced tumor extent $(\geq \mathrm{pT} 3)$ than in cases of limited tumor extent $(\leq \mathrm{pT} 2)\left(P<0.001, \chi^{2}\right)$, we hypothesized if this unequal distribution may contribute to its negative prognostic effect. However, multivariate Cox regression, including pT status, Gleason score, and residual status, indicated that KDM5C expression remained an independent prognostic parameter $(P=0.002)$ (Table 3$)$.

Interestingly, the prognostic power of nuclear KDM5C expression could be highlighted by stratifying the survival
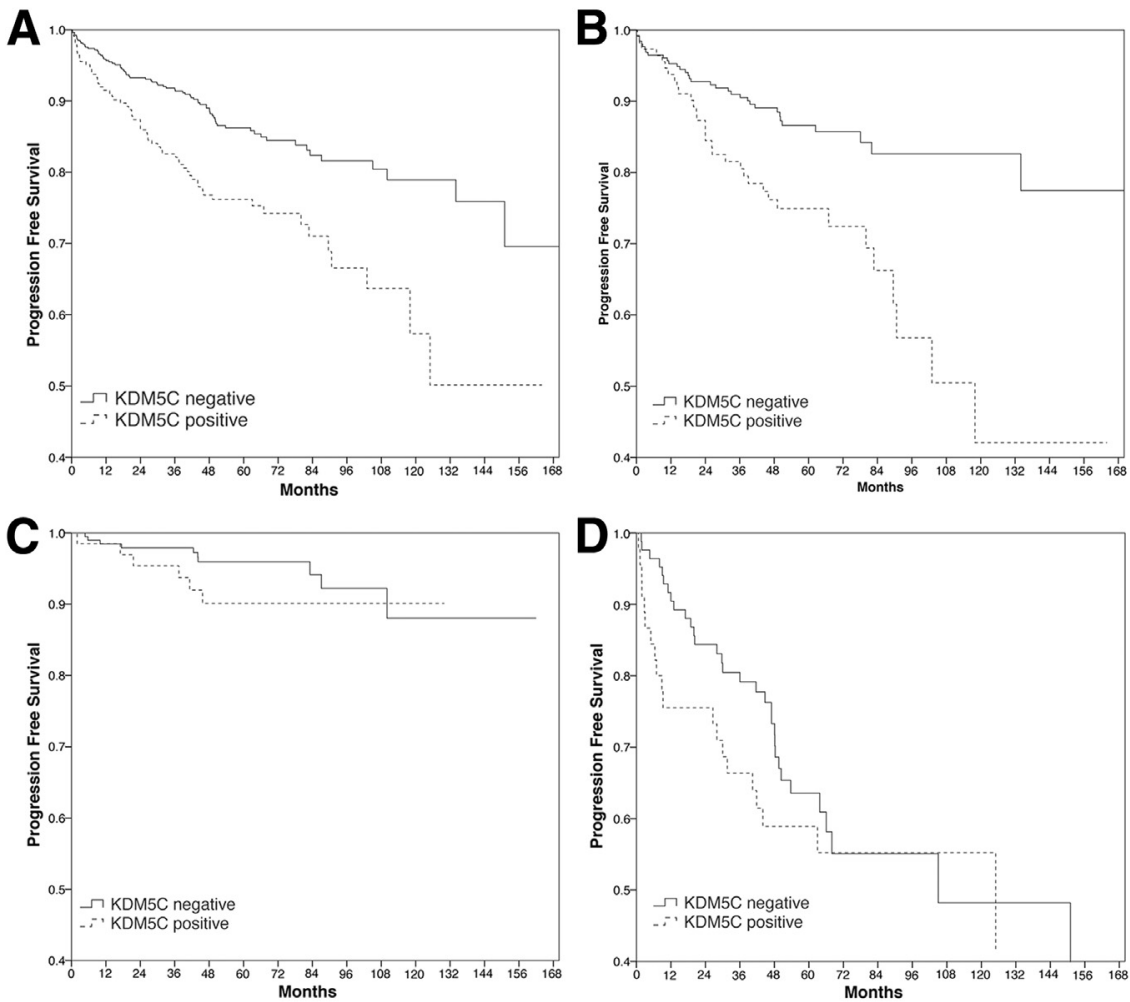

Figure 2 A: Kaplan-Meier survival analysis. Kaplan-Meier analysis of 761 prostate tumors shows that nuclear KDM5C expression is associated with shorter survival of patients with PCa. KaplanMeier analysis comparing PFS of nuclear KDM5C stratified according to the Gleason groups 7 (B), $<7$ (C), and $>7$ (D). In this model the prognostic effect is most pronounced for the Gleason group 7 (group 7, $P<0.001$; group $<7, P=0.273$; group $>7, P=0.308) . n=367(B), n=264$ (C), and $n=130$ (D). $P<0.001($ A). 
Table 3 Multivariate Survival Analysis (Cox Regression) Regarding PSA Relapse-Free Survival after Prostatectomy

\begin{tabular}{|c|c|c|c|c|c|c|c|c|c|}
\hline \multirow[b]{2}{*}{ Parameter } & \multicolumn{3}{|c|}{ Bonn cohort } & \multicolumn{3}{|c|}{ Berlin cohort } & \multicolumn{3}{|l|}{ All } \\
\hline & $P$ value & $\operatorname{Exp}(B)$ & CI $95 \%$ & $P$ value & $\operatorname{Exp}(B)$ & CI $95 \%$ & $P$ value & $\operatorname{Exp}(B)$ & CI $95 \%$ \\
\hline KDM5C & 0.017 & 2.095 & $1.138-3.855$ & 0.025 & 1.619 & $1.061-2.472$ & 0.002 & 1.733 & $1.226-2.450$ \\
\hline Gleason score & $<0.001$ & 3.095 & $1.891-5.065$ & 0.001 & 1.717 & $1.244-2.370$ & $<0.001$ & 2.061 & $1.580-2.689$ \\
\hline pT status & 0.473 & 1.263 & $0.667-2.392$ & $<0.001$ & 2.695 & $1.642-4.425$ & $<0.001$ & 2.045 & $1.389-2.308$ \\
\hline
\end{tabular}

$\operatorname{Exp}(B)$, hazard ratio; KDM5C, lysine demethylase 5C; PSA, prostate-specific antigen; $p T$, pathological stage; $R$, residual.

analyses for Gleason groups $<7,7$, and $>7$. In this model the prognostic effect was most pronounced for the Gleason group 7 (group 7, $P<0.001$; group $<7, P=0.273$; group $>7, P=0.308$ ) (Figure 2, B-D). Next, we asked if KDMC5 expression was a prognostic indicator for Gleason score 7 , when Gleason score 7 adenocarcinomas are split into $3+4=7$ and $4+3=7$ subgroups. In both Gleason score 7 subgroups KDM5C was a prognostic indicator and reached significance in the larger clinically important $3+4$ group (Supplemental Figure S1).

\section{Inhibition of KDM5C Impairs Cell Growth in Vitro}

We used human prostate cell lines to evaluate the potential functional roles of $\mathrm{KDM} 5 \mathrm{C}$ in vitro. We first examined KDM5C expression in both cell lines of prostatic cancer (PC3, LNCAP, and Du145) and a benign human hyperplastic prostatic epithelial cell. LNCaP cells are androgen-sensitive human prostate adenocarcinoma cells derived from a lymph node metastasis. DU145 cell line was derived from brain metastasis, and PC3 cell lines were established from bone metastasis. Both cell lines did not respond to androgens. PC3 cells have high metastatic potential compared with DU145 cells that have a moderate metastatic potential and compared with LNCaP cells that have low metastatic potential. We observed only low KDM5C expression in the benign prostatic hyperplastic cell line and moderate expression in LNCaP cells, whereas we observed high levels of KDM5C in DU145 and PC3 cells (Figure 3A), indicating a positive correlation between androgen-resistant malignancy and KDM5C expression. The role of KDM5C in proliferation of $\mathrm{PCa}$ cells was assessed after knockdown of KDM5C expression with the use of siRNAs. PC3 cells were transiently transfected with two distinct siRNAs directed against KDM5C or with a scrambled control siRNA. Significant KDM5C knockdown was detected 6 days after transfection by qPCR (Figure 3B). Knockdown dramatically reduced cell viability in PC3 cells 6 days after transfection (Figure 3C). Decreased viability was accompanied by down-regulation of proliferation-associated genes such as MKI67 and PCNA and induction of p21 (Figure 3D), suggesting that KDM5C is required for proliferation in this cell model.

\section{Discussion}

This is the first comprehensive description of expression patterns of KDM5C in large cohorts of clinically organconfined PCas after radical prostatectomy. Our results show that KDM5C expression, as determined by immunohistochemistry, is a promising prognostic factor for biochemical recurrence after radical prostatectomy and thus a predictive candidate marker for therapy failure. This prognostic value
A

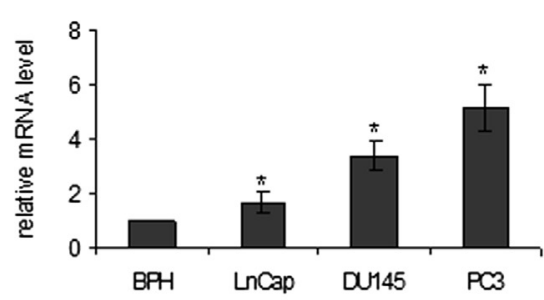

C

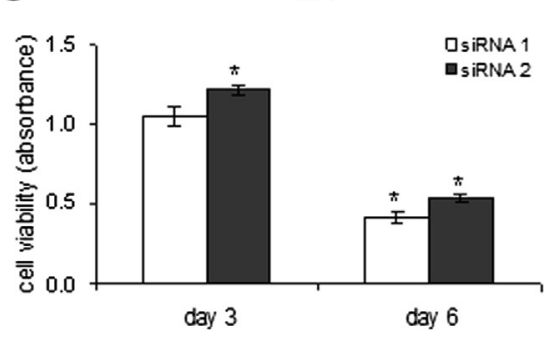

B

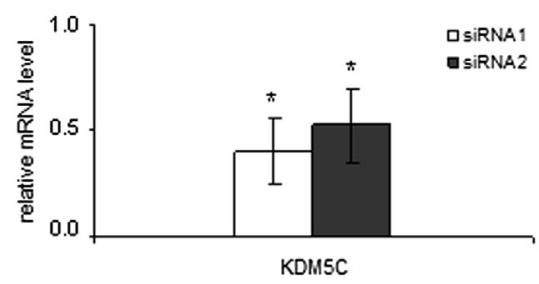

D

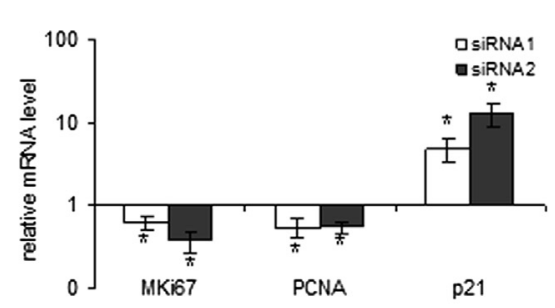

Figure 3 Inhibition of $\mathrm{KDM5C}$ impairs $\mathrm{PCa}$ growth in vitro. A: Confirmation of differential expression of $\mathrm{KDM} 5 \mathrm{C}$ in prostate cell lines by using real-time PCR analysis. B: $q P C R$ analysis to determine the knockdown of LSD1 mRNA in PC3 cells 6 days after transfection. C: MTT assay of PC3 cells treated with two different siRNAs against KDM5C detects a significant reduction in cell number after 6 days of incubation. D: Knockdown of $\mathrm{KDMSC}$ induces down-regulation of proliferation-associated genes such as MKI67 and PCNA and induction of p21. qPCR analysis was done in $\mathrm{PC} 3$ cells with two siRNAs directed against KDM5C or with scrambled control siRNA. In all experiments, 18S rRNA was used as the endogenous reference gene. Data are expressed as fold change to scrambled siRNA transfected samples which were set to 1 (B-D). ${ }^{*} P<0.05$. 
is particularly pronounced in the common and clinically important subgroup of patients with Gleason score 7.

Notably, PCa biology varies from locally confined tumors with low risk of relapse to tumors with high risk of progression even after radical prostatectomy. Because of the PSA screening the detection rate of frequently indolent $\mathrm{PCa}$ has increased in recent years, but, so far, reliable biomarkers to predict tumor relapse and poor clinical outcome are still missing. The exploration of the pathogenic roles of KDM5C may open new avenues for an improved oncologic risk assessment. Although the therapeutic procedure is relatively clearly defined both for well-differentiated and poorly differentiated carcinomas, the therapeutic decision between radical prostatectomy and nonsurgical treatment approaches (such as active surveillance, radiation, or antihormonal therapy) is particularly challenging in the subgroup of moderately differentiated carcinomas. In this context, an immunohistochemical risk profile for cancer treatment decision could facilitate therapeutic stratification. As to whether the prognostic KDM5C effect is also applicable as a preoperative risk factor, additional studies are needed that can focus on the biopsy state, in which patients are divided into different surgical and nonsurgical treatment groups. Patient groups for such a prospective evaluation are currently being established as part of the multicenter Evaluation of Four Treatment Modalities in Prostate Cancer with Low or Early Intermediate Risk study. ${ }^{20}$

In addition, various molecular points of KDM5C may serve as a pathogenically relevant biomarker. Similar to genetic alterations, epigenetic aberrations contribute significantly to tumor initiation and progression. Recent evidence indicates that members of the KDM5/JARID cluster may also be involved in PCa metastasis. KDM5B, which is significantly overexpressed in local and metastatic $\mathrm{PCa}$, is an androgen receptor coactivator and may play important roles in controlling the invasion and metastasis of PCa cells. ${ }^{21}$

One of the primary hallmarks of cancer is its limitless replicative ability, much of which is acquired through overexpression of oncogenes and repression of tumor suppressor genes. KDM5C is capable of removing the $\mathrm{H} 3 \mathrm{~K} 4 \mathrm{me} 3-$ activating mark from histones, which makes KDM5C a potential player in the down-regulation of tumor suppressors and suggests that KDM5C activity could repress oncogenes. Our data clearly show that in vitro silencing of KDM5C by an RNA interference approach leads to induction of p21 and subsequent inhibition of cellular proliferation. p21 belongs to the cyclin-dependent kinase interacting protein/kinase inhibitory protein family of cyclin-dependent kinase inhibitors, and it inhibits proliferation mainly by interfering with cyclin E/cyclin-dependent kinase 2 activity. ${ }^{22}$ In addition, silencing of KDM5C was shown to inhibit tumor cell growth by down-regulating genes involved in proliferation such as PCNA and MKI67. KDM5C contributes to cell proliferation through regulation of cell cycle-regulatory genes. This finding suggests that down-regulation leads to suppression of tumor formation, which makes KDM5C an ideal drug target for PCa. In addition, KDM5C was shown to inhibit the transcriptional activity of SMAD3, a transforming growth factor $\beta$-dependent transcription factor, independently of its enzymatic activity. ${ }^{23}$ The transforming growth factor $\beta / S M A D$ signaling cascade works with the phosphatidylinositol 3-kinase/Akt signaling cascade to ensure PCa cell survival and protection against apoptosis. Transforming growth factor $\beta$ signaling in PCa blocks early tumorigenesis but paradoxically enhances metastatic spreading. ${ }^{24}$

Preclinical studies suggest that inhibition of histonemodifying enzymes can suppress tumorigenesis and provide a strong rationale for development of their inhibitors for use in cancer therapy. One class of histone-modifying enzymes that has been successfully targeted for cancer therapy is the family of histone deacetylases (HDACs). Many histone demethylases, including $\mathrm{KDM} 5 \mathrm{~A},{ }^{25} \mathrm{KDM} 5 \mathrm{~B},{ }^{21,26}$ and $\mathrm{KDM} 5 \mathrm{C},{ }^{27}$ have been shown to interact with the HDAC complexes. These studies suggest that success of HDAC inhibitors could be correlated to their interactions with the KDM5 enzymes. HDACs generally act on many different histone residues, whereas the catalytic activity of the KDM enzymes is limited to specific histone residues. This suggests that KDM inhibitors are likely to have more specific biological effects and therefore be more specific anticancer epidrugs than HDAC inhibitors. Recently, a structurally unique small molecule that specifically inhibits the activity of the Jumonji family of histone demethylases in vitro in cancer cells and in tumors in vivo was identified. Unlike known inhibitors, this inhibitor is not a competitive inhibitor of $\alpha$-ketoglutarate. It was shown that the new inhibitor alters a subset of transcriptional pathways and blocks viability in cancer but not in patient-matched normal cells. ${ }^{28}$

In summary, we provide the first evidence that KDM5C is overexpressed in $\mathrm{PCa}$, associated with poor prognosis, and may serve as a novel drug target in PCa.

\section{Acknowledgments}

We thank Susanne Steiner and Anke Röper for excellent technical assistance.

\section{Supplemental Data}

Supplemental material for this article can be found at http://dx.doi.org/10.1016/j.ajpath.2014.05.022.

\section{References}

1. Siegel R, Naishadham D, Jemal A: Cancer statistics, 2013. CA Cancer J Clin 2013, 63:11-30

2. Dal Pra A, Cury FL, Souhami L: Radiation therapy and androgen deprivation in the management of high risk prostate cancer. Int Braz J Urol 2011, 37:161-175. discussion 176-179

3. Pezaro C, Attard G: Prostate cancer in 2011: redefining the therapeutic landscape for CRPC. Nat Rev Urol 2012, 9:63-64 
4. Saad F, Hotte SJ: Guidelines for the management of castrate-resistant prostate cancer. Can Urol Assoc J 2010, 4:380-384

5. Attard G, de Bono JS: Translating scientific advancement into clinical benefit for castration-resistant prostate cancer patients. Clin Cancer Res 2011, 17:3867-3875

6. Shi Y, Lan F, Matson C, Mulligan P, Whetstine JR, Cole PA, Casero RA, Shi Y: Histone demethylation mediated by the nuclear amine oxidase homolog LSD1. Cell 2004, 119:941-953

7. Metzger E, Wissmann M, Yin N, Müller JM, Schneider R, Peters AH, Günther T, Buettner R, Schüle R: LSD1 demethylates repressive histone marks to promote androgen-receptor-dependent transcription. Nature 2005, 437:436-439

8. Metzger E, Imhof A, Patel D, Kahl P, Hoffmeyer K, Friedrichs N, Müller JM, Greschik H, Kirfel J, Ji S, Kunowska N, BeisenherzHuss C, Günther T, Buettner R, Schüle R: Phosphorylation of histone H3T6 by PKCbeta(I) controls demethylation at histone H3K4. Nature 2010, 464:792-796

9. Tsukada Y, Fang J, Erdjument-Bromage H, Warren ME, Borchers CH, Tempst P, Zhang Y: Histone demethylation by a family of JmjC domain-containing proteins. Nature 2006, 439:811-816

10. Lin W, Cao J, Liu J, Beshiri ML, Fujiwara Y, Francis J, Cherniack AD, Geisen C, Blair LP, Zou MR, Shen X, Kawamori D, Liu Z, Grisanzio C, Watanabe H, Minamishima YA, Zhang Q, Kulkarni RN, Signoretti S, Rodig SJ, Bronson RT, Orkin SH, Tuck DP, Benevolenskaya EV, Meyerson M, Kaelin WG Jr, Yan Q: Loss of the retinoblastoma binding protein 2 (RBP2) histone demethylase suppresses tumorigenesis in mice lacking Rb1 or Men1. Proc Natl Acad Sci U S A 2011, 108:13379-13386

11. Blair LP, Cao J, Zou MR, Sayegh J, Yan Q: Epigenetic regulation by lysine demethylase 5 (KDM5) enzymes in cancer. Cancers 2011, 3: 1383-1404

12. Varier RA, Outchkourov NS, de Graaf P, van Schaik FM, Ensing HJ, Wang F, Higgins JM, Kops GJ, Timmers HT: A phospho/methyl switch at histone $\mathrm{H} 3$ regulates TFIID association with mitotic chromosomes. EMBO J 2010, 29:3967-3978

13. Roesch A, Fukunaga-Kalabis M, Schmidt EC, Zabierowski SE, Brafford PA, Vultur A, Basu D, Gimotty P, Vogt T, Herlyn M: A temporarily distinct subpopulation of slow-cycling melanoma cells is required for continuous tumor growth. Cell 2010, 141:583-594

14. Sharma SV, Lee DY, Li B, Quinlan MP, Takahashi F, Maheswaran S, McDermott U, Azizian N, Zou L, Fischbach MA, Wong KK, Brandstetter K, Wittner B, Ramaswamy S, Classon M, Settleman J: A chromatin-mediated reversible drug-tolerant state in cancer cell subpopulations. Cell 2010, 141:69-80

15. Iwase S, Lan F, Bayliss P, de la Torre-Ubieta L, Huarte M, Qi HH, Whetstine JR, Bonni A, Roberts TM, Shi Y: The X-linked mental retardation gene SMCX/JARID1C defines a family of histone H3 lysine 4 demethylases. Cell 2007, 128:1077-1088
16. Dalgliesh GL, Furge K, Greenman C, Chen L, Bignell G, Butler A, et al: Systematic sequencing of renal carcinoma reveals inactivation of histone modifying genes. Nature 2010, 463:360-363

17. Epstein JI, Allsbrook WC Jr, Amin MB, Egevad LL; ISUP Grading Committee: The 2005 International Society of Urological Pathology (ISUP) Consensus Conference on Gleason Grading of Prostatic Carcinoma. Am J Surg Pathol 2005, 29:1228-1242

18. Liang Z, Diamond M, Smith JA, Schnell M, Daniel R: Proliferating cell nuclear antigen is required for loading of the SMCX/KMD5C histone demethylase onto chromatin. Epigenetics Chromatin 2011, 4:18

19. Lim S, Janzer A, Becker A, Zimmer A, Schüle R, Buettner R, Kirfel J: Lysine-specific demethylase 1 (LSD1) is highly expressed in ERnegative breast cancers and a biomarker predicting aggressive biology. Carcinogenesis 2010, 31:512-520

20. Kristiansen G, Stöckle M, Albers P, Schmidberger H, Martus P, Wellek S, Härter M, Bussar-Maatz R, Wiegel T: [The importance of pathology in the German prostate cancer study PREFERE] German. Pathologe 2013, 34:449-462

21. Xiang Y, Zhu Z, Han G, Ye X, Xu B, Peng Z, Ma Y, Yu Y, Lin H, Chen AP, Chen CD: JARID1B is a histone H3 lysine 4 demethylase up-regulated in prostate cancer. Proc Natl Acad Sci U S A 2007, 104: 19226-19231

22. Abbas T, Dutta A: p21 in cancer: intricate networks and multiple activities. Nat Rev Cancer 2009, 9:400-414

23. Kim TD, Shin S, Janknecht R: Repression of Smad3 activity by histone demethylase SMCX/JARID1C. Biochem Biophys Res Commun 2008, 366:563-567

24. Buijs JT, Henriquez NV, van Overveld PG, van der Horst G, ten Dijke P, van der Pluijm G: TGF-beta and BMP7 interactions in tumour progression and bone metastasis. Clin Exp Metastasis 2007, 24:609-617

25. Klose RJ, Yan Q, Tothova Z, Yamane K, Erdjument-Bromage H, Tempst P, Gilliland DG, Zhang Y, Kaelin WG Jr: The retinoblastoma binding protein RBP2 is an H3K4 demethylase. Cell 2007, 128: 889-900

26. Barrett A, Santangelo S, Tan K, Catchpole S, Roberts K, SpencerDene B, Hall D, Scibetta A, Burchell J, Verdin E, Freemont P, TaylorPapadimitriou J: Breast cancer associated transcriptional repressor PLU-1/JARID1B interacts directly with histone deacetylases. Int J Cancer 2007, 121:265-275

27. Huang PH, Chen CH, Chou CC, Sargeant AM, Kulp SK, Teng CM, Byrd JC, Chen CS: Histone deacetylase inhibitors stimulate histone H3 lysine 4 methylation in part via transcriptional repression of histone $\mathrm{H} 3$ lysine 4 demethylases. Mol Pharmacol 2011, 79:197-206

28. Wang L, Chang J, Varghese D, Dellinger M, Kumar S, Best AM, Ruiz J, Bruick R, Peña-Llopis S, Xu J, Babinski DJ, Frantz DE, Brekken RA, Quinn AM, Simeonov A, Easmon J, Martinez ED: A small molecule modulates Jumonji histone demethylase activity and selectively inhibits cancer growth. Nat Commun 2013, 4:2035 